\title{
Regulating cardiac energy metabolism and bioenergetics by targeting the DNA damage repair protein BRCA1
}

\author{
Krishna K. Singh, PhD, ${ }^{\text {a,b }}$ Praphulla C. Shukla, PhD, ${ }^{\text {a,b }}$ Bobby Yanagawa, MD, PhD,,${ }^{\text {a,b }}$ \\ Adrian Quan, MPhil, ${ }^{\mathrm{a}, \mathrm{b}}$ Fina Lovren, PhD,${ }^{\mathrm{a}, \mathrm{b}}$ Yi Pan, MD, ${ }^{\mathrm{a}, \mathrm{b}}$ Cory S. Wagg, ${ }^{\mathrm{c}}$ Hwee Teoh, PhD, ${ }^{\mathrm{a}, \mathrm{b}, \mathrm{d}}$ \\ Gary D. Lopaschuk, PhD, ${ }^{c}$ and Subodh Verma, MD, PhD, FRCSC ${ }^{\mathrm{a}, \mathrm{b}}$
}

\begin{abstract}
Objective: Alterations in cardiac energy and substrate metabolism play a critical role in the development and clinical course of heart failure. We hypothesized that the cardioprotective role of the breast cancer 1, early onset (BRCA1) gene might be mediated in part by alterations in cardiac bioenergetics.
\end{abstract}

\begin{abstract}
Methods: We generated cardiomyocyte-specific BRCA1 homozygous and heterozygous knockout mice using the Cre-loxP technology and evaluated the key molecules and pathways involved in glucose metabolism, fatty acid metabolism, and mitochondrial bioenergetics.

Results: Cardiomyocyte-specific BRCA1-deficient mice showed reduced cardiac expression of glucose and fatty acid transporters, reduced acetyl-coenzyme A carboxylase 2 and malonyl-coenzyme A decarboxylase (key enzymes that control malonyl coenzyme A, which in turn controls fatty acid oxidation), and reduced carnitine palmitoyltransferase I, a rate-limiting enzyme for mitochondrial fatty acid uptake. Peroxisome proliferator-activated receptor $\alpha$ and $\gamma$ and carnitine palmitoyltransferase I levels were also downregulated in these hearts. Rates of glucose and fatty acid oxidation were reduced in the hearts of heterozygous cardiomyocyte-restricted BRCA1-deficient mice, resulting in a decrease in the rate of adenosine triphosphate production. This decrease in metabolism and adenosine triphosphate production occurred despite an increase in 5'-adenosine monophosphate-activated protein kinase and AKT activation in the heart.
\end{abstract}

Conclusions: Cardiomyocyte-specific loss of BRCA1 alters critical pathways of fatty acid and glucose metabolism, leading to an energy starved heart. BRCA1-based cell or gene therapy might serve as a novel target to improve cardiac bioenergetics in patients with heart failure. (J Thorac Cardiovasc Surg 2013;146:702-9)

Germline mutations in the tumor suppressor breast cancer 1, early onset (BRCA1) gene are highly penetrant for an increased risk of breast and ovarian cancer syndromes. ${ }^{1}$ BRCA1 functions as a key gatekeeper of genome stability, with an established role in key cellular processes involved in DNA damage repair, transcriptional regulation, ubiquitination, cell cycle control, and cellular regeneration. ${ }^{2}$

We have recently reported that BRCA1 and BRCA2 are not only critical tumor suppressors but also function as

From the Divisions of Cardiac Surgery ${ }^{\mathrm{a}}$ and Endocrinology and Metabolism, ${ }^{\mathrm{d}}$ and the

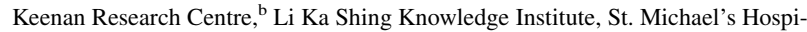
tal, University of Toronto, Toronto, Ontario, Canada; and Cardiovascular Research Centre, ${ }^{\mathrm{c}}$ Mazankowski Alberta Heart Institute, University of Alberta, Edmonton, Alberta, Canada

This work was supported by grants from the Heart and Stroke Foundation of Canada and the Canadian Institutes of Health Research (to S.V.). B.Y. was the recipient of a Physicians' Services Incorporated Foundation Resident Research Grant. S.V. is the Canada Research Chair in Atherosclerosis at the University of Toronto.

Disclosures: Authors have nothing to disclose with regard to commercial support.

K.K.S, P.C.S., and B.Y. share the position of first author.

Received for publication Sept 24, 2012; revisions received Nov 15, 2012; accepted for publication Dec 11, 2012; available ahead of print Jan 14, 2013

Address for reprints: Subodh Verma, MD, PhD, FRCSC, Division of Cardiac Surgery, Department of Surgery, University of Toronto, St. Michael's Hospital, Suite 8-003, Bond Wing, 30 Bond St, Toronto, ON M5B 1W8, Canada (E-mail: vermasu@smh.ca).

0022-5223/\$36.00

Copyright (c) 2013 by The American Association for Thoracic Surgery

http://dx.doi.org/10.1016/j.jtcvs.2012.12.046 key gatekeepers of cardiac structure and function, particularly in the setting of ischemic, genotoxic, or septic stressors. $^{3-5}$ Using a Cre-loxP system, we generated BRCA1 and BRCA2 cardiomyocyte-specific (CM) knockout mice and demonstrated that experimental myocardial infarction or genotoxic stress in the form of doxorubicin elicited profound cardiac failure and death. ${ }^{3,4}$ Mechanistically, we demonstrated that decreased repair or accumulation of damaged DNA led to increased cardiomyocyte apoptosis by a p53-dependent pathway in these mice. ${ }^{3-5}$ These data suggest that BRCA1 might be a previously unrecognized essential regulator of cardiac function and that BRCA1-based therapies could serve as a novel therapeutic avenue for ischemic and genotoxic heart failure.

In addition to cardiomyocyte apoptosis, alterations in cardiac energy and substrate metabolism play a critical role in the development and eventual transition into cardiac failure. ${ }^{6}$ Since it has been suggested that BRCA1 interacts with acetyl-coenzyme A (CoA) carboxylase (ACC) and inhibits fatty acid synthesis in cancer cells, ${ }^{7}$ we hypothesized that BRCA1 might, in addition to regulating apoptosis, serve to regulate cardiac bioenergetics. In the present report, we specifically evaluated the key molecules involved in fatty acid and glucose metabolism in the heart and provide evidence that BRCA1 deficiency results in an energy-compromised myocardium, primarily through 


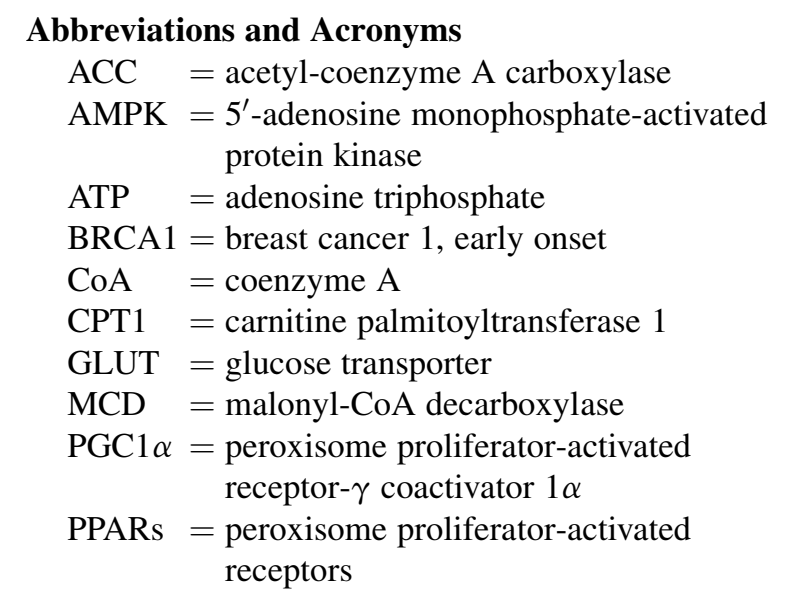

a global downregulation of myocardial carbohydrate and fatty acid metabolism.

\section{METHODS}

\section{Animal Studies}

All animal procedures were performed in accordance with the guidelines of the Canadian Council on Animal Care and were approved by the St. Michael's Hospital Animal Care Committee. To directly elucidate the role of BRCA1 on cardiac structure, function, and survival and to circumvent the embryonic lethality associated with systemic loss of BRCA $1,{ }^{8}$ we applied the Cre-loxP system to disrupt BRCA1 expression specifically in the cardiomyocytes. ${ }^{3}$ Conditional inactivation of BRCA1 in the cardiomyocytes was achieved by crossing mice homozygous for the exon 11 floxed BRCA1 allele with hemizygous mice expressing Cre-recombinase under the control of the $\alpha$-myosin heavy chain promoter, which is predominantly expressed in the adult cardiomyocyte. ${ }^{9}$ The mice, regardless of their genotypes, were viable and fertile and were born in the expected Mendelian ratios. Analysis of genomic DNA from the heart, brain, liver, and skeletal muscles revealed that the Cre-mediated recombined allele was only present in the heart samples and absent in the brain, liver, and skeletal muscles of $\mathrm{CM}-\mathrm{BRCA} 1^{+-}$and $\mathrm{CM}-\mathrm{BRCA} 1^{-1-}$ mice. Successful deletion of BRCA1 at the protein level was confirmed by Western blot and immunohistochemical analysis. Taken together, these findings validate the successful generation of mice with cardiomyocyte-specific inactivation of full-length BRCA1. ${ }^{3}$

\section{RNA Measurements and Western Blotting}

Total whole heart RNA was reverse transcribed and real-time reactions performed with a SYBR Green master mix on the PRISM 7900HT system (both Life Technologies, Carlsbad, Calif).

The immunoblots were probed with antibodies from Cell Signaling (Beverley, Mass) against ACC, phosphorylated ACC, AKT, phosphorylated AKT, 5'-adenosine monophosphate-activated protein kinase (AMPK), phosphorylated AMPK, and peroxisome proliferator-activated receptor- $\gamma$ coactivator $1 \alpha(\mathrm{PGCl} \alpha)$ or from Chemicon (Billerica, Mass) directed against glyceraldehyde 3-phosphate dehydrogenase. Immunoband signals were visualized by enhanced chemiluminescence (GE Healthcare Biosciences, Pittsburgh, Pa).

\section{Fatty Acid and Glucose Metabolism}

Ex vivo working mice hearts were aerobically perfused, as previously described. ${ }^{10}$ The rates of glucose and palmitate oxidation were measured directly from the production of ${ }^{14} \mathrm{CO}_{2}$ and ${ }^{3} \mathrm{H}_{2} \mathrm{O}$ from $\left[\mathrm{U}_{-}{ }^{14} \mathrm{C}\right]$ glucose and $\left[9,10-{ }^{3} \mathrm{H}\right]$ palmitate, respectively. ${ }^{10}$ The adenosine triphosphate (ATP) yield was calculated using a stoichiometric ratio of $30 \mathrm{~mol} \mathrm{ATP} / \mathrm{mol}$ glucose and $105 \mathrm{~mol} \mathrm{ATP} / \mathrm{mol}$ palmitate being oxidized. ${ }^{10}$ This calculation assumes $100 \%$ coupling of mitochondrial oxidative phosphorylation.

\section{Statistical Analysis}

Unless otherwise stated, the data are presented as the mean \pm SD. Realtime data were analyzed using the relative expression software tool. The Student $t$ test was applied when data from 2 groups were compared. Differences between multiple groups were evaluated using analysis of variance, with the Bonferroni correction.

\section{RESULTS}

\section{Cardiomyocyte-Specific Deletion of BRCA1 Reduces Acetyl-CoA Carboxylase 2 and Malonyl-CoA Decarboxylase Expression}

In addition to being a critical regulator of the lipogenic pathway, ACC is a key regulator of fatty acid oxidation in the heart, secondary to catalyzing the carboxylation of acetyl-CoA to form malonyl-CoA, a potent inhibitor of mitochondrial fatty acid uptake. ${ }^{11,12}$ Compared with their wild-type control littermates, CM-BRCA $1^{-1-}$ and CM-BRCA $1^{+/-}$mice demonstrated a significant reduction in cardiac expression of $\mathrm{ACC} 2$, the predominant cardiac variant of ACC, but not ACC1 (Figure 1, $A$ and $B$ ). Total ACC levels were indistinguishable between the groups (data not shown). Malonyl-CoA decarboxylase (MCD) acts to regulate malonyl-CoA levels through its decarboxylation of malonyl-CoA back into acetyl-CoA, thereby stimulating fatty acid oxidation. ${ }^{13}$ CM- BRCA1 loss resulted in a reduction in cardiac MCD expression (Figure 1, C).

\section{Cardiomyocyte-Specific Deletion of BRCA1 Alters Peroxisome Proliferator-Activated Receptor Signaling}

The peroxisome proliferator-activated receptors (PPARs) are a family of ligand-activated transcription factors within the broad nuclear receptor superfamily. ${ }^{14} \operatorname{PPAR} \alpha$, a regulator of myocardial lipid and energy metabolism, was significantly downregulated in the hearts of CM-BRCA $1^{+/-}$and CM-BRCA1 ${ }^{-\prime-}$ mice (Figure 2, A). ${ }^{15}$ However, PPAR $\beta$ expression was similar across the groups (Figure $2, B$ ). In contrast, the levels of cardioprotective PPAR $\gamma$ were also significantly downregulated in BRCA1-deficient hearts (Figure 2, C).

Differential regulation was observed in several important downstream PPAR substrates. ACC2 and MCD are regulated by $\operatorname{PPAR} \alpha$, and both were downregulated in the hearts of CM-BRCA $1^{+/-}$and CM-BRCA $1^{-1-}$ mice (Figure 1, $B$ and $C$ ), as was the membrane-associated 88-kDa fatty acid translocase CD36, a mediator of cardiac fatty acid uptake (Figure $3, A) .{ }^{16}$ Glucose transporter 

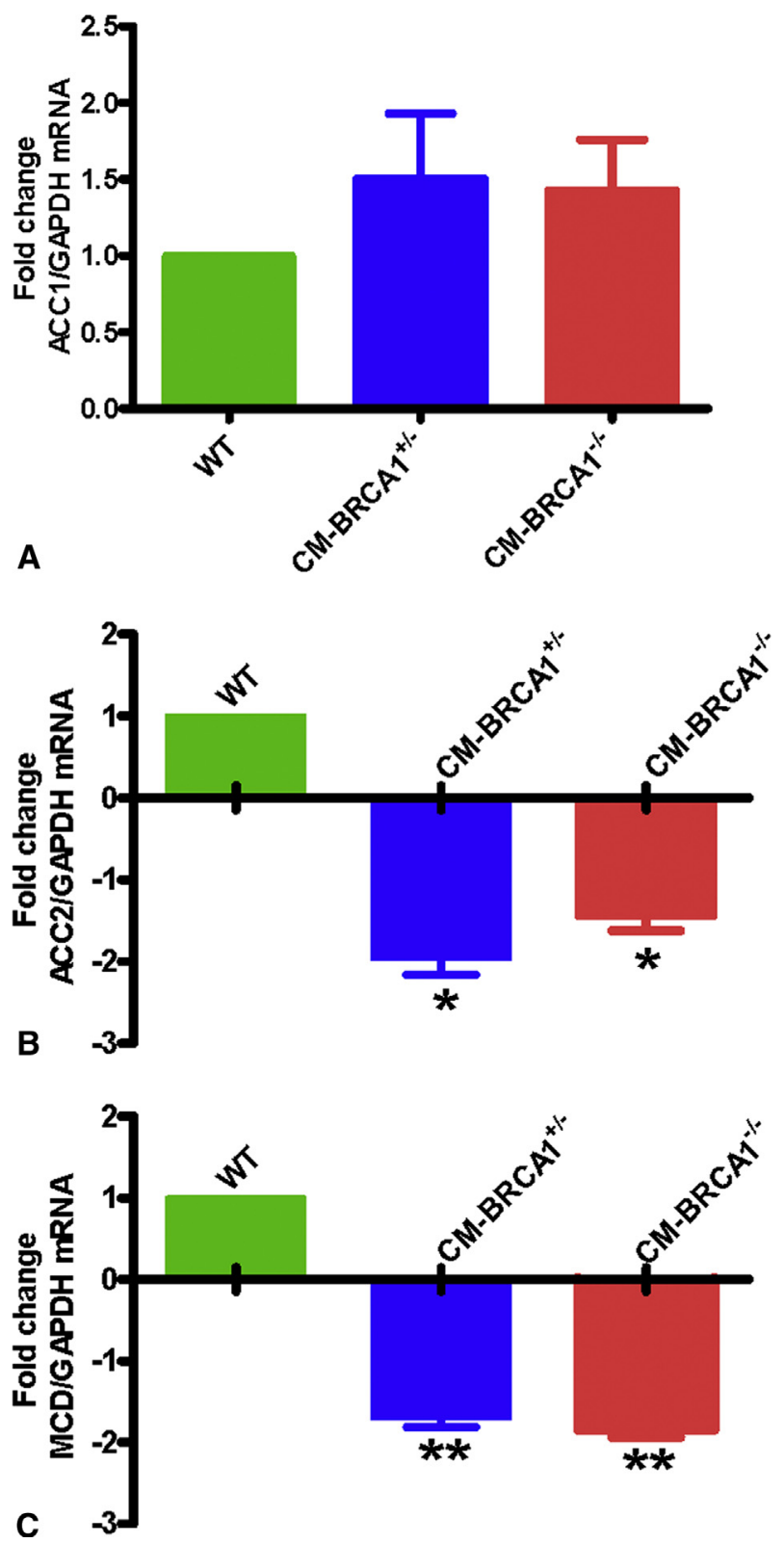

FIGURE 1. Cardiomyocyte-specific ( $C M$ ) deletion of the breast cancer 1, early onset (BRCA1) gene reduces cardiac acetyl-coenzyme A carboxylase $(A C C) 2$ and malonyl-CoA decarboxylase $(M C D)$ expression. Total RNA and protein were extracted from the hearts of 32-week-old female $\mathrm{CM}_{-\mathrm{BRCA}}{ }^{+/-}, \mathrm{CM}^{-\mathrm{BRCA}^{-/-}}{ }^{-}$, and wild-type $(W T)$ control littermates. Real-time polymerase chain reaction was performed for A, ACC1, B, ACC2 and C, MCD. $\mathrm{n}=3$ /group. $* P<.05, * * P<.01$ versus WT.

(GLUT)-4, but not GLUT-1, was downregulated in the hearts of $\mathrm{CM}-\mathrm{BRCA} 1^{+-}$and $\mathrm{CM}-\mathrm{BRCA}^{-1-}$ mice (Figure 3, $B$ and $C$ ). Carnitine palmitoyltransferase 1 (CPT1), a PPAR-responsive gene and the rate-limiting enzyme in the mitochondrial uptake of free fatty acids, was also downregulated in BRCA1-deficient hearts (Figure 3, D). ${ }^{17}$
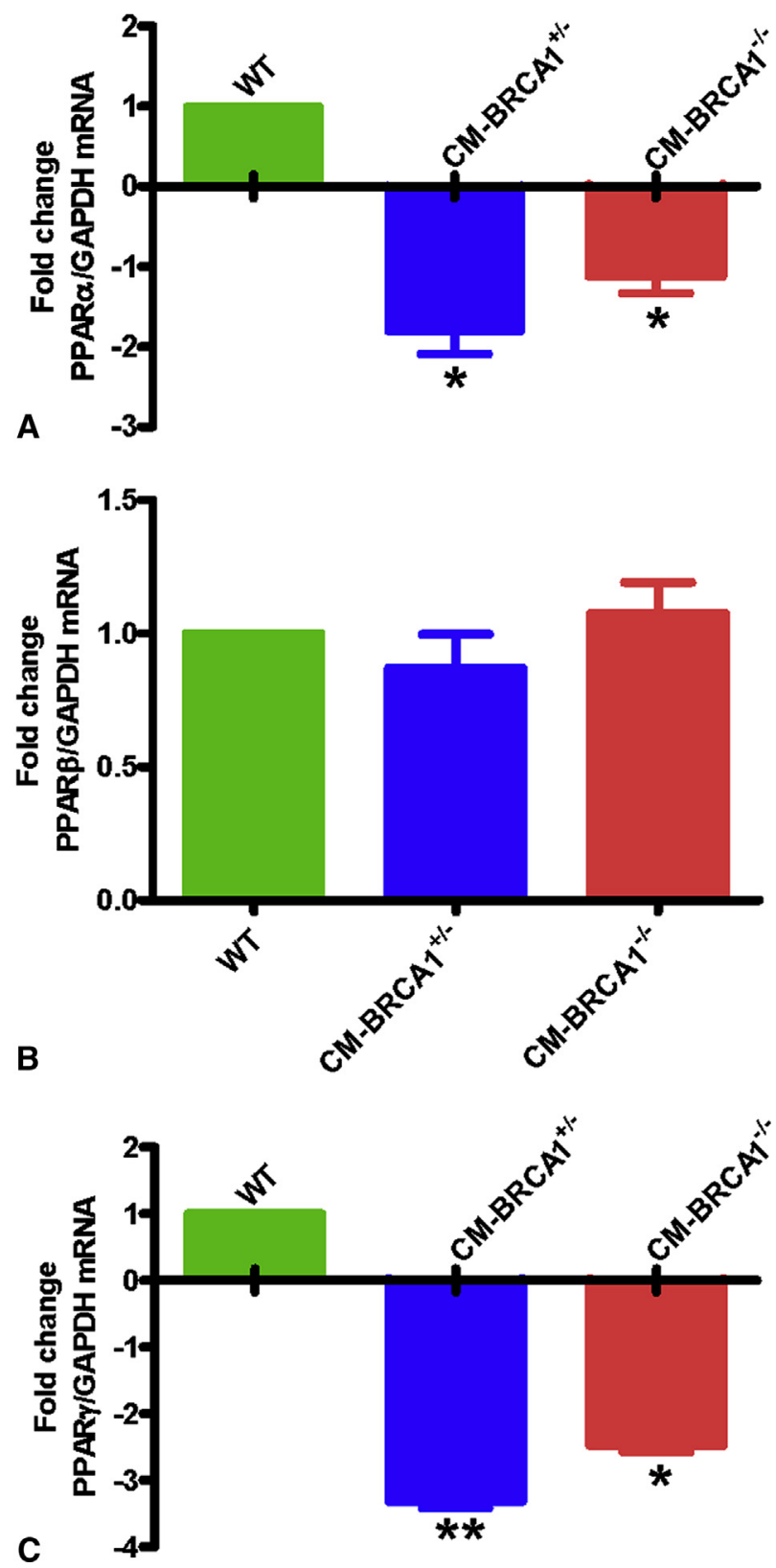

FIGURE 2. Cardiomyocyte-specific (CM) deletion of the breast cancer 1, early onset (BRCA1) gene alters peroxisome proliferator-activated receptor $(P P A R)$ signaling in the heart. Total RNA was extracted from the hearts of 32-week-old female CM-BRCA $1^{+/-}, \mathrm{CM}^{\mathrm{BRCA}} 1^{-/-}$, and wild-type (WT) control littermates. Real-time polymerase chain reaction was performed for $\mathrm{A}, \operatorname{PPAR} \alpha, \mathrm{B}, \operatorname{PPAR} \beta$, and $\mathrm{C}, \operatorname{PPAR} \gamma . \mathrm{n}=3$ /group. ${ }^{*} P<.05, * * P<.01$ versus WT.

\section{Cardiomyocyte-Specific Deletion of BRCA1} Activates AMPK and AKT and Reduces PPAR Coactivator-1 $\alpha$ Expression

Activation of AMPK, a stress response triggering myocardial glucose uptake and glycolysis, in addition to phosphorylating ACC and increasing fatty acid oxidation, 

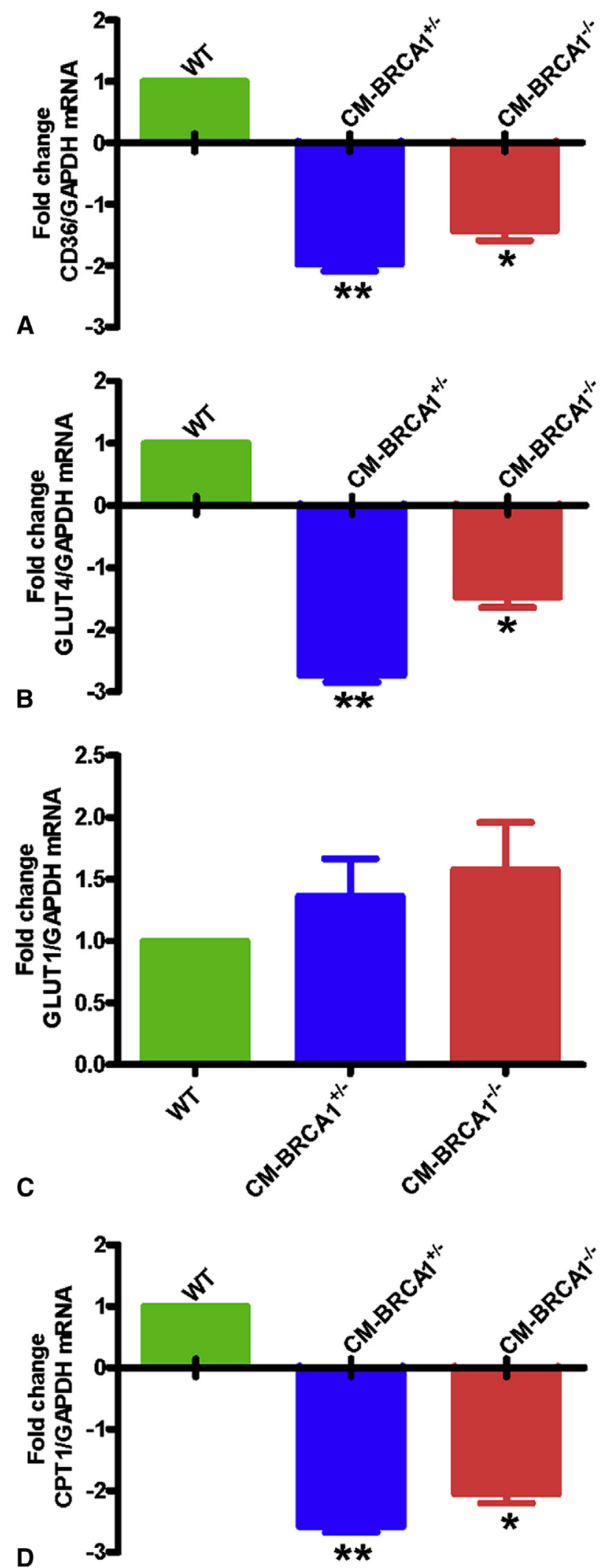

FIGURE 3. Cardiomyocyte $(C M)$-specific deletion of the breast cancer 1, early onset (BRCA1) gene reduces induction of peroxisome was increased in the hearts of $\mathrm{CM}-\mathrm{BRCA} 1^{+/-}$and CM-BRCA $1^{-/-}$mice (Figure $\left.4, A\right) .{ }^{18}$ Similarly, consistent with its role in metabolic stress responses, phosphorylated AKT levels were increased (Figure 4, B). In contrast, the levels of PGC $1 \alpha$, a master activator of mitochondrial biogenesis that is reduced in failing hearts, ${ }^{19}$ were reduced in the hearts of the CM-BRCA $1^{+/-}$and $\mathrm{CM}-\mathrm{BRCA} 1^{-/-}$ mice (Figure 4, C).

\section{Cardiomyocyte-Specific Deletion of BRCA1 Reduces Glucose and Fatty Acid Oxidation Rates and ATP Production in the Heart}

The glucose oxidation and palmitate oxidation rates in mouse hearts perfused in the working mode were measured, and the ATP production rates from these 2 pathways were calculated (Figure 5). Glucose oxidation (Figure 5, $A$ ) and fatty acid oxidation (Figure $5, B$ ) were significantly suppressed in hearts from the more clinically relevant BRCA1-haploinsufficient CM-BRCA1 ${ }^{+/-}$mice than in the hearts from the wild-type control littermates. This resulted in a significant decrease in the ATP production rates from glucose and palmitate oxidation in BRCA1-deficient hearts compared with the hearts of wild-type control littermates (Figure 5, C).

\section{DISCUSSION}

The heart has a very high energy demand, and alterations in cardiac energy production contribute to the severity of ischemic injury and the development of heart failure. ${ }^{20} \mathrm{We}$ have previously demonstrated a cardioprotective role of BRCA $1 / 2$ in response to ischemic and genotoxic stresses. ${ }^{3,4}$ Although we had proposed that the primary protective mechanism through which BRCA1 acts involves DNA damage repair and the prevention of cardiomyocyte apoptosis, our present results suggest that BRCA1 might also play an important role in regulating cardiac energy and substrate metabolism. Accordingly, we suggest that a loss of BRCA1 leads to a reduction in the cardiac energy supply, with downregulation of both fatty acid and glucose metabolic pathways and mitochondrial bioenergetics.

BRCA1 is a critical molecule of inherited breast and ovarian cancer syndromes. The tumor metabolic microenvironment is described by the Warburg phenomenon, the preferential shift to glycolysis from glucose oxidation, even in

proliferator-activated receptor $(P P A R)$ targets in the heart. Total RNA was extracted from the hearts of 32 -week-old female CM-BRCA1 ${ }^{+/-}$, CM-BRCA $1^{-/-}$, and wild-type (WT) control littermates. Real-time polymerase chain reaction was performed for A, CD36, B, glucose transporter (GLUT)-4, C, GLUT-1, and D, carnitine palmitoyltransferase 1 (CPT1). $\mathrm{n}=3$ /group. $* P<.05, * * P<.01$ versus $\mathrm{WT}$. 

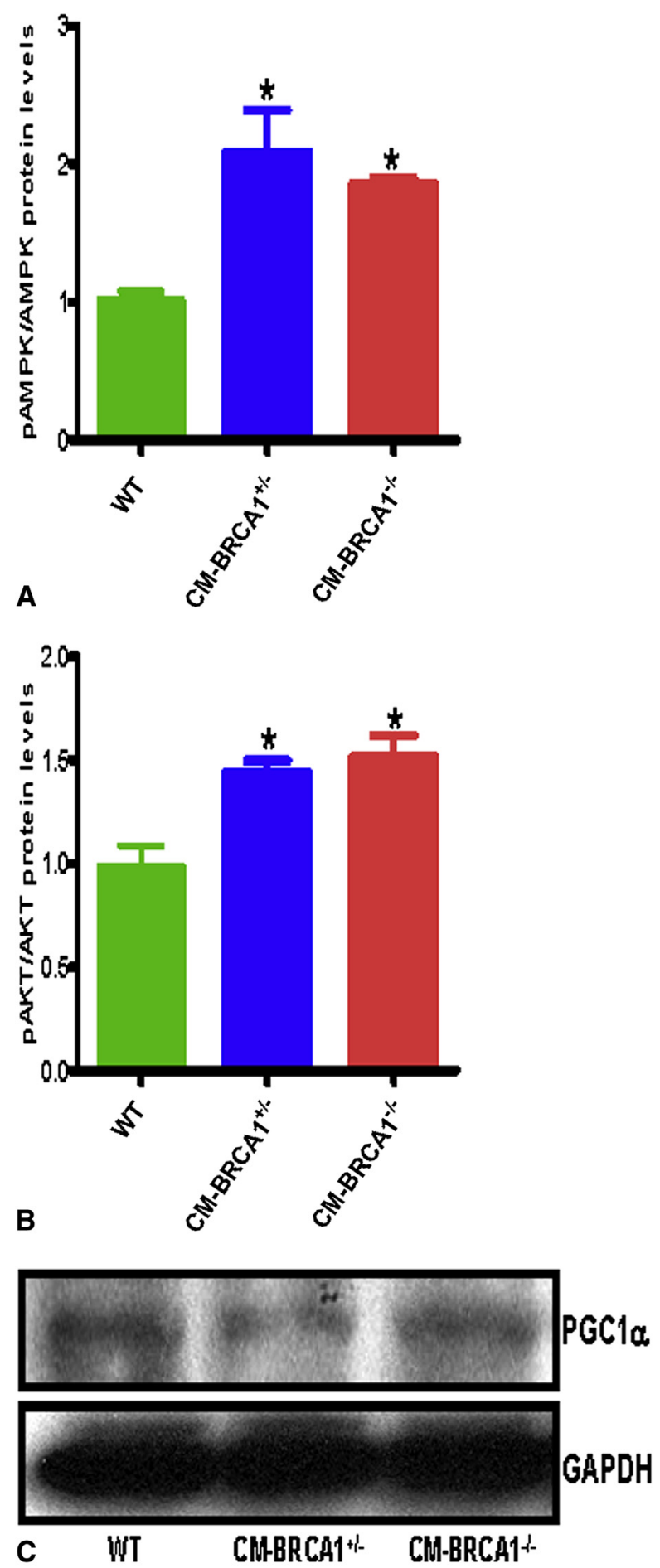

FIGURE 4. Cardiomyocyte-specific ( $C M)$ deletion of the breast cancer 1, early onset (BRCAl) gene activates cardiac 5'-adenosine monophosphateactivated protein kinase $(A M P K)$ and AKT, while reducing peroxisome proliferator-activated receptor- $\gamma$ coactivator $1 \alpha(P G C 1 \alpha)$ expression. Total protein was extracted from the hearts of 32-week-old female CM$\mathrm{BRCA}^{+/-}, \mathrm{CM}-\mathrm{BRCA}^{-/-}$, and wild-type $(W T)$ control littermates. Western blots were performed and quantified for A, total and phosphorylated $5^{\prime}$-adenosine monophosphate-activated protein kinase (AMPK), oxygen-rich environments, coupled with the stimulation of fatty acid synthesis. ${ }^{21}$ The BRCA1 C-terminal domain directly binds and sequesters $\mathrm{ACC} 1{ }^{21}$ Hence, mutations in BRCA1 disrupt the BRCA1-ACC1 complex, freeing ACC1, which results in unregulated fatty acid synthesis, essential for breast cancer proliferation. ${ }^{21}$ However, the broad distribution of BRCA1 in tissues, including cardiac tissue, suggests important roles for BRCA1-metabolic regulation outside of tumor inhibition. ${ }^{3}$

Cardiac metabolism of fatty acid and carbohydrates is finely regulated to provide a continuous source of energy in the form of ATP, necessary to sustain contractile activity and other cellular functions. ${ }^{6,22}$ Alterations in cardiac bioenergetics have been associated with human and experimental cardiac pathologic states. ${ }^{6,23,24}$ In ischemic hearts, glucose oxidation and glycolysis are uncoupled, and fatty acid metabolism predominates, resulting in impaired functional recovery, progressive ventricular remodeling, and eventual cardiac failure. ${ }^{24}$ We found in BRCA1-deficient hearts, downregulation of enzymes that control both glucose and fatty acid oxidation, instead of a shift from one to the other. Substrate transporters within the sarcolemma are a key regulatory component of myocardial glucose and fatty acid use, ${ }^{25}$ and we found downregulation of both the glucose transporter GLUT-4 and the fatty acid transporter CD36 in BRCA1-deficient hearts. In BRCA1-deficient hearts, MCD (which degrades malonylCoA, an inhibitor of CPT1), was also downregulated, blocking fatty acid oxidation. ${ }^{13}$ The PPAR $\alpha$-responsive gene CPT1, a rate-limiting enzyme in the mitochondrial uptake of fatty acids, was also downregulated. ${ }^{17,26}$ The combined decrease in CD36, MCD, and CPT1 expression could all potentially contribute to the decrease in fatty acid oxidation observed in the BRCA1-deficient hearts.

An important finding in the present work was the decrease in PPARs, a family of ligand-activated transcription factors within the broad nuclear receptor superfamily. $\operatorname{PPAR} \alpha$, abundant in tissues with high rates of mitochondrial fatty acid oxidation, such as myocardial tissue, regulates the target genes involved in cellular lipid catabolism. ${ }^{15}$ In contrast, PPAR $\gamma$ directs the expression of genes involved in fat storage, and recent studies have suggested that PPAR $\gamma$ agonists are cardioprotective against an ischemic insult. ${ }^{27,28} \mathrm{We}$ report significant downregulation of $\operatorname{PPAR} \alpha$ and PPAR $\gamma$ in BRCA1-deficient hearts. This suggests dysregulation of both glucose and fatty acid oxidation. We observed coincident downregulation of CD36, the PPAR-responsive membrane-associated $88-\mathrm{kDa}$ fatty acid translocase that controls cardiac fatty acid uptake and GLUT- $4 .^{29,30}$

\footnotetext{
$\mathrm{B}$, total and phosphorylated AKT, and $\mathrm{C}, \mathrm{PGC} 1 \alpha$. Glyceraldehyde 3-phosphate dehydrogenase $(G A P D H)$ was used as a loading control. $\mathrm{n}=3-4$ /group. $* P<.05$ versus WT.
} 

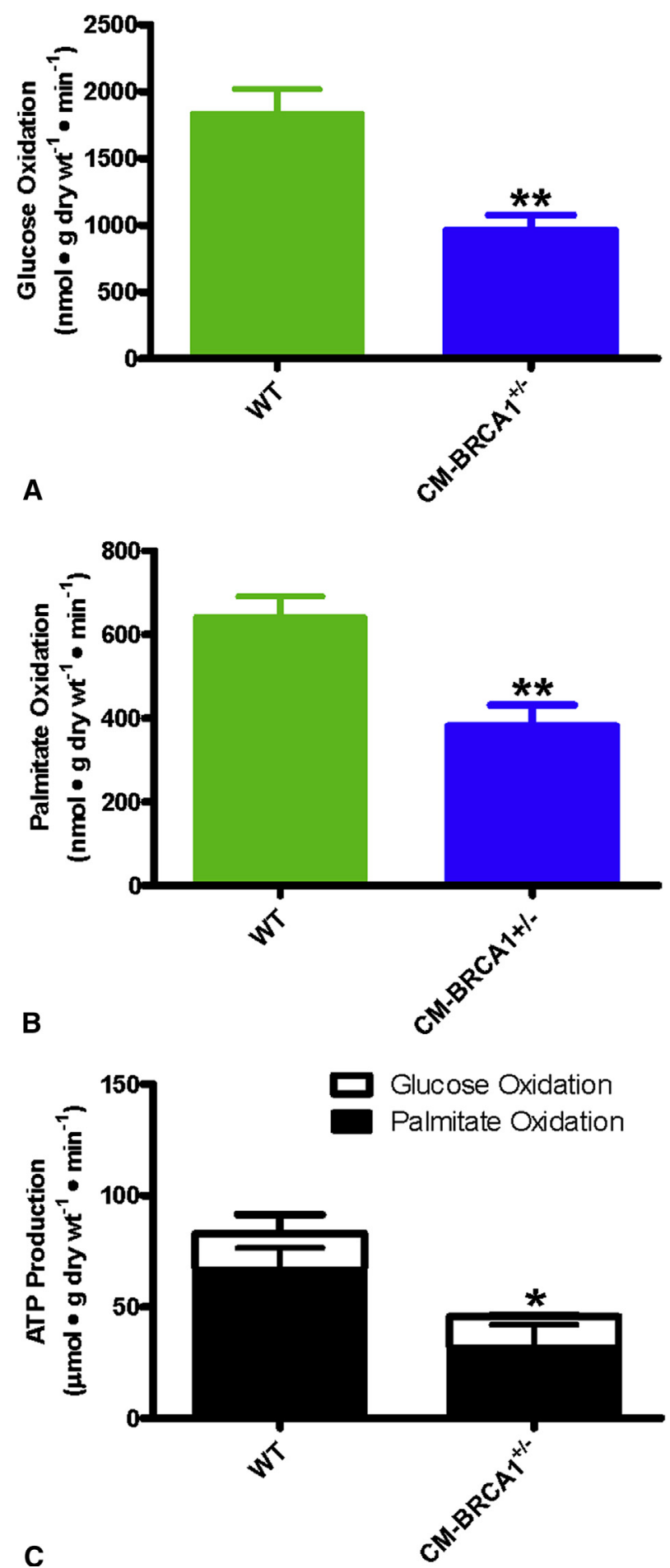

FIGURE 5. Cardiomyocyte-specific ( $C M$ ) deletion of the breast cancer 1, early onset (BRCA1) gene reduces rates of glucose and fatty acid oxidation and adenosine triphosphate (ATP) production in the heart. Rates of A, glucose oxidation, B, palmitate oxidation and $\mathrm{C}$, ATP production were determined in the aerobic hearts of CM-BRCA1 ${ }^{+/-}$and wild-type $(W T)$ control littermates ex vivo. $\mathrm{n}=4$-6/group. $* P<.05, * * P<.01$ versus WT.

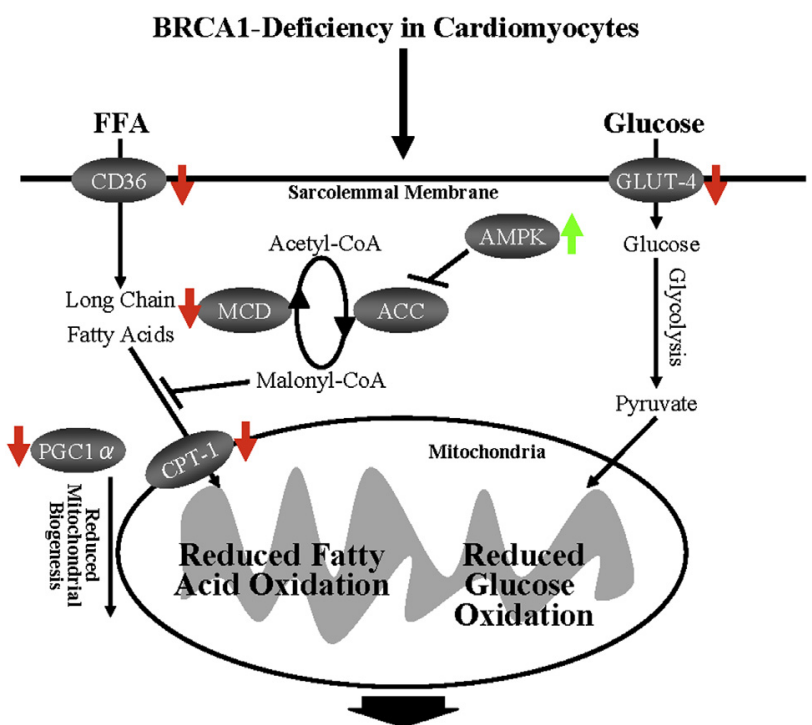

Global Myocardial Metabolic Depression

FIGURE 6. Proposed mechanism for breast cancer 1, early onset (BRCA1) gene-mediated metabolic regulation. Fatty acid and glucose transporters are reduced in BRCA1-deficient cardiomyocytes, leading to reduced $\beta$-oxidation and glucose oxidation. Acetyl-coenzyme A $(\mathrm{CoA})$ carboxylase $(A C C)$ carboxylates acetyl-CoA into malonyl-CoA, and malonylCoA decarboxylase $(M C D)$ decarboxylates malonyl-CoA back into acetylCoA. MCD is reduced in BRCA1-deficient cardiomyocytes, causing malonyl-CoA to accumulate, which in turn leads to inhibited mitochondrial uptake of fatty acids through carnitine palmitoyltransferase 1 (CPT1). CPT1 levels, which are also reduced in BRCA1-deficient cardiomyocytes, result in inhibition of $\beta$-oxidation rates. $5^{\prime}$-Adenosine monophosphateactivated protein kinase $(A M P K)$ phosphorylation negatively regulates $\mathrm{ACC}$, and mitochondrial biogenesis is reduced owing to decreases in PGC1 $\alpha$ levels. In brief, cardiomyocyte-specific loss of BRCA1 results in reduced expression of fatty acid and glucose transporters, reduced levels of downstream mediators of fatty acid and glucose oxidation, and reduced mitochondrial biogenesis, leading to an energy starved heart and eventually culminating in heart failure. All downregulated and upregulated molecules are shown by red and green arrows, respectively. FFA, Free fatty acid.

The key stress-induced metabolic regulator AMPK, which promotes glucose uptake and glycolysis and fatty acid oxidation, was upregulated in BRCA1-deficient hearts. AMPK is triggered by cardiac ischemia, ${ }^{31}$ and the increase in AMPK levels might have resulted from attempts to increase energy supply. This is consistent with experimental severe end-stage heart failure, demonstrating depression in the overall oxidative metabolism, with a preserved ratio between fatty acid and glucose oxidation. ${ }^{32}$ AKT, which promotes glucose oxidation by enhancing glucose uptake through glucose transporters and attenuates fatty acid oxidation through downregulation of $\operatorname{PPAR} \alpha$ and $\operatorname{PGC} 1 \alpha$, was also upregulated in BRCA1-deficient hearts, which again might have been an attempted compensator response to the metabolic stress in these hearts. ${ }^{18}$

The rates of flux through metabolic pathways are controlled by the extent of expression of key metabolic 
molecules (eg, enzymes and transporters) and complex metabolic pathway regulation. ${ }^{6}$ The reduced levels of fatty acid and glucose transporters in BRCA1-deficient cardiomyocytes were associated with less $\beta$-oxidation and glucose oxidation. Significantly reduced ATP production rates were also noted in BRCA1-deficient hearts. We therefore propose that BRCA1 regulates metabolic control by the schema illustrated in Figure 6. ACC carboxylates acetylCoA into malonyl-CoA and MCD decarboxylates malonyl-CoA back into acetyl-CoA. MCD is reduced in BRCA1-deficient cardiomyocytes, causing malonyl-CoA to accumulate, which in turn leads to inhibited mitochondrial uptake of fatty acids through CPT1. CPT1 levels, which are also reduced in BRCA1-deficient cardiomyocytes, result in inhibition of $\beta$-oxidation rates. Also, AMPK phosphorylation negatively regulates ACC, and mitochondrial biogenesis is reduced owing to decreases in PGC1 $\alpha$ levels. In brief, cardiomyocyte-specific loss of BRCA1 results in the reduced expression of fatty acid and glucose transporters, reduced levels of downstream mediators of fatty acid and glucose oxidation, and reduced mitochondrial biogenesis, leading to an energy starved heart, eventually culminating in heart failure.

Using a lentivirus system, Niwano and colleagues ${ }^{33}$ developed a sarcoplasmic/endoplasmic reticulum $\mathrm{Ca}^{2+}$-ATPase 2 gene transfer system and successfully demonstrated integration of the sarcoplasmic/endoplasmic reticulum $\mathrm{Ca}^{2+}$-ATPase 2 gene into the rat heart, which resulted in beneficial cardiac remodeling, function, and survival outcomes after induction of myocardial infarction-associated heart failure. ${ }^{33}$ Recently, investigators of the phase 2 Calcium Upregulation by Percutaneous Administration of Gene Therapy in Cardiac Disease study reported that patients with advanced heart failure who received intracoronary sarcoplasmic/endoplasmic reticulum $\mathrm{Ca}^{2+}$-ATPase $2 \mathrm{a}$ gene therapy based on an adeno-associated virus 1 system exhibited either improved or stabilized heart failure symptoms, exercise tolerance, serum biomarker levels, and left ventricular function. ${ }^{34}$ These studies, although preliminary, are encouraging and by no means minor advancements in the field. Future studies aimed at using direct intramyocardial delivery of adenoviral, adeno-associated virus, or lentiviral gene delivery approaches in larger animal models (eg, porcine) could be envisioned. BRCA1 might represent a novel platform for cell-based approaches to myocardial regeneration, and BRCA1-overexpressing bone marrow cells, myoblasts, or progenitor cells might provide an alternative cell therapy mode.

The present study had a few limitations. Although we have previously demonstrated impaired left ventricular systolic function and increased mortality rates in CM-BRCA1-deficient mice in response to ischemic and genotoxic stressors, the present study would have been strengthened if the glucose oxidation and fatty acid oxidation rates were measured simultaneous as a function of generated cardiac power or $\mathrm{dP} / \mathrm{dT}$ was measured using, for example, a Langendorff preparation. However, we did assess the rates of glucose oxidation and palmitate oxidation in mouse hearts perfused in the "working heart mode," and the ATP production rates from these 2 pathways were calculated. We also acknowledge that the study would have been improved further by a gain of function approach using either viral delivery of BRCA1 or cell-based therapy to determine whether function and metabolism could be improved.

\section{References}

1. Foulkes WD. Inherited susceptibility to common cancers. N Engl J Med. 2008; 359:2143-53.

2. Venkitaraman AR. Cancer susceptibility and the functions of BRCA1 and BRCA2. Cell. 2002;108:171-82.

3. Shukla PC, Singh KK, Quan A, Al-Omran M, Teoh H, Lovren F, et al. BRCA1 is an essential regulator of heart function and survival following myocardial infarction. Nat Commun. 2011;2:593.

4. Singh KK, Shukla PC, Quan A, Desjardins JF, Lovren F, Pan Y, et al. BRCA2 protein deficiency exaggerates doxorubicin-induced cardiomyocyte apoptosis and cardiac failure. J Biol Chem. 2012;287:6604-14.

5. Teoh H, Quan A, Creighton AK, Bang AKW, Singh KK, Shukla PC, et al. BRCA1 gene therapy reduces systemic inflammatory response and multiple organ failure and improves survival in experimental sepsis. Gene Ther. Epub. 2012 Jan 19.

6. Stanley WC, Recchia FA, Lopaschuk GD. Myocardial substrate metabolism in the normal and failing heart. Physiol Rev. 2005;85:1093-129.

7. Moreau K, Dizin E, Ray H, Luquain C, Lefai E, Foufelle F, et al. BRCA1 affects lipid synthesis through its interaction with acetyl-CoA carboxylase. J Biol Chem. 2006;281:3172-81.

8. Hakem R, de la Pompa JL, Mak TW. Developmental studies of BRCA1 and BRCA2 knock-out mice. J Mammary Gland Biol Neoplasia. 1998;3:431-45.

9. Agah R, Frenkel PA, French BA, Michael LH, Overbeek PA, Schneider MD. Gene recombination in postmitotic cells: targeted expression of Cre recombinase provokes cardiac-restricted, site-specific rearrangement in adult ventricular muscle in vivo. J Clin Invest. 1997;100:169-79.

10. Belke DD, Larsen TS, Lopaschuk GD, Severson DL. Glucose and fatty acid metabolism in the isolated working mouse heart. Am J Physiol. 1999;277:R1210-7.

11. Mabrouk GM, Helmy IM, Thampy KG, Wakil SJ. Acute hormonal control of acetyl-CoA carboxylase: the roles of insulin, glucagon, and epinephrine. J Biol Chem. 1990;265:6330-8.

12. Wakil SJ, Titchener EB, Gibson DM. Evidence for the participation of biotin in the enzymic synthesis of fatty acids. Biochim Biophys Acta. 1958;29:225-6.

13. Cuthbert KD, Dyck JR. Malonyl-CoA decarboxylase is a major regulator of myocardial fatty acid oxidation. Curr Hypertens Rep. 2005;7:407-11.

14. Desvergne B, Wahli W. Peroxisome proliferator-activated receptors: nuclear control of metabolism. Endocr Rev. 1999;20:649-88.

15. Barger PM, Kelly DP. PPAR signaling in the control of cardiac energy metabolism. Trends Cardiovasc Med. 2000;10:238-45.

16. van der Vusse GJ, Glatz JF, Stam HC, Reneman RS. Fatty acid homeostasis in the normoxic and ischemic heart. Physiol Rev. 1992;72:881-940.

17. Ussher JR, Lopaschuk GD. The malonyl CoA axis as a potential target for treating ischaemic heart disease. Cardiovasc Res. 2008;79:259-68.

18. Lopaschuk GD. AMP-activated protein kinase control of energy metabolism in the ischemic heart. Int J Obes (Lond). 2008;32(suppl 4):S29-35.

19. Faerber G, Barreto-Perreia F, Schoepe M, Gilsbach R, Schrepper A, Schwarzer M, et al. Induction of heart failure by minimally invasive aortic constriction in mice: reduced peroxisome proliferator-activated receptor gamma coactivator levels and mitochondrial dysfunction. J Thorac Cardiovasc Surg. 2011; 141:492-500.e1.

20. Lopaschuk GD, Ussher JR, Folmes CD, Jaswal JS, Stanley WC. Myocardial fatty acid metabolism in health and disease. Physiol Rev. 2010;90:207-58.

21. Magnard C, Bachelier R, Vincent A, Jaquinod M, Kieffer S, Lenoir GM, et al. BRCA1 interacts with acetyl-CoA carboxylase through its tandem of BRCT domains. Oncogene. 2002;21:6729-39. 
22. Starling RC, Hammer DF, Altschuld RA. Human myocardial ATP content and in vivo contractile function. Mol Cell Biochem. 1998;180:171-7.

23. Ingwall JS, Weiss RG. Is the failing heart energy starved? On using chemical energy to support cardiac function. Circ Res. 2004;95:135-45.

24. Taha M, Lopaschuk GD. Alterations in energy metabolism in cardiomyopathies. Ann Med. 2007;39:594-607.

25. Glatz JF, Bonen A, Ouwens DM, Luiken JJ. Regulation of sarcolemmal transport of substrates in the healthy and diseased heart. Cardiovasc Drugs Ther. 2006;20: 471-6.

26. Folmes CD, Lopaschuk GD. Role of malonyl-CoA in heart disease and the hypothalamic control of obesity. Cardiovasc Res. 2007;73:278-87.

27. Yue TL. Cardioprotective effects of thiazolidinediones, peroxisome proliferatoractivated receptor-gamma agonists. Drugs Today (Barc). 2003;39:949-60.

28. Zhu P, Lu L, Xu Y, Schwartz GG. Troglitazone improves recovery of left ventricular function after regional ischemia in pigs. Circulation. 2000;101:1165-71.

29. Luiken JJ, Willems J, van der Vusse GJ, Glatz JF. Electrostimulation enhances FAT/CD36-mediated long-chain fatty acid uptake by isolated rat cardiac myocytes. Am J Physiol Endocrinol Metab. 2001;281:E704-12.
30. Wood IS, Trayhurn P. Glucose transporters (GLUT and SGLT): expanded families of sugar transport proteins. Br J Nutr. 2003;89:3-9.

31. Kudo N, Barr AJ, Barr RL, Desai S, Lopaschuk GD. High rates of fatty acid oxidation during reperfusion of ischemic hearts are associated with a decrease in malonyl-CoA levels due to an increase in $5^{\prime}$-AMP-activated protein kinase inhibition of acetyl-CoA carboxylase. J Biol Chem. 1995;270:17513-20.

32. Doenst T, Pytel G, Schrepper A, Amorim P, Farber G, Shingu Y, et al. Decreased rates of substrate oxidation ex vivo predict the onset of heart failure and contractile dysfunction in rats with pressure overload. Cardiovasc Res. 2010;86:461-70.

33. Niwano K, Arai M, Koitabashi N, Watanabe A, Ikeda Y, Miyoshi H, et al. Lentiviral vector-mediated SERCA2 gene transfer protects against heart failure and left ventricular remodeling after myocardial infarction in rats. Mol Ther. 2008 16:1026-32.

34. Jessup M, Greenberg B, Mancini D, Cappola T, Pauly DF, Jaski B, et al. Calcium Upregulation by Percutaneous Administration of Gene Therapy in Cardiac Disease (CUPID): a phase 2 trial of intracoronary gene therapy of sarcoplasmic reticulum $\mathrm{Ca}^{2+}$-ATPase in patients with advanced heart failure. Circulation. 2011;124:304-13. 